\title{
Docetaxel-loaded Nanopharmaceutical CRLX301
}

National Cancer Institute

\section{Source}

National Cancer Institute. Docetaxel-loaded Nanopharmaceutical CRLX301. NCI

Thesaurus. Code C121539.

A nanoparticle-based formulation containing the poorly water-soluble, secondgeneration taxane analog docetaxel, with antineoplastic activity. Upon intravenous administration of the docetaxel-loaded nanopharmaceutical CRLX301, the nanoparticles are able to accumulate at the tumor site due to the unique characteristics of the tumor's vasculature, while avoiding normal, healthy tissue. In turn, CRLX301 is taken up by the tumor cell via macropinocytosis. Subsequently, docetaxel is slowly released into the cytoplasm where it binds to and stabilizes the beta-subunit of tubulin, thereby stabilizing microtubules and inhibiting microtubule disassembly. This prevents mitosis and results in cell death. Compared to the administration of docetaxel alone, this formulation is able to increase docetaxel's efficacy while avoiding systemic exposure, which minimizes its toxicity. 